\title{
Suddenly fixed upward ocular deviation under general anesthesia
}

\author{
Won Jae Kim ${ }^{1}$, Sang-Jin Park ${ }^{2}$, Myung Mi Kim ${ }^{1}$ \\ Departments of ${ }^{1}$ Ophthalmology and ${ }^{2}$ Anesthesiology and Pain Medicine, Yeungnam University College of Medicine, Daegu, Korea
}

Various changes in ocular position are possible during general anesthesia as opposed to the awakening state. However, unexpected ocular deviation under general anesthesia is a disconcerting event as it can lead to difficult complications intraoperatively. To date, sudden fixed upward ocular deviation has been rarely reported previously. This phenomenon was observed in an 8-year-old boy during strabismus surgery. Suddenly fixed upward ocular deviation occurred when the speculum was inserted into the right eye. When the eyeball was pulled down, using forceps, there was some resistance, such as contracture of superior rectus. The eyeball sprang back into the upward position when the forceps was released. These changes could hamper the good exposition of the surgical field, leading to significant intraoperative difficulties and complications. Surgeons should be aware of this possibility, despite general anesthesia; if it occurs, proceed with the surgery as planned preoperatively, and both ophthalmic and anesthetic interventions should be used to solve this problem.

Keywords: Strabismus; General anesthesia; Muscle relaxant

\section{INTRODUCTION}

Previous studies reported changes in ocular position during general anesthesia, and some attempted to evaluate the use of these changes in ocular position to improve surgical outcomes [1-4]. Moreover, others reported different changes in the ocular position (fixed, eccentrically upward deviation) under general anesthesia [5]. These sudden unexpected ocular deviations during strabismus surgery would be disconcerting for many surgeons as it could hamper good exposition of the surgical field, leading to significant intraoperative difficulties and complications. However, to date, there has been limited reports of this ocular deviation in the literature. Hence, we

Received: March 6, 2017, Revised: March 27, 2017

Accepted: April 4, 2017

Corresponding Author: Myung Mi Kim, Department of Ophthalmology, Yeungnam University College of Medicine,

170 Hyeonchung-ro, Nam-gu, Daegu 42415, Korea

Tel: +82-53-620-3441, Fax: +82-53-626-5936

E-mail: mmk@med.yu.ac.kr reported this suddenly fixed upward ocular deviation in an 8 -year-old boy during strabismus surgery under general anesthesia.

\section{CASE}

Informed consent was obtained from the parents of our patient. An 8-year-old boy was referred to our clinic for exotropia and chin elevation. He had no systemic disease. He demonstrated 35 prism diopters (PD) exotropia in the primary position, with $2+$ overaction of inferior oblique (IO) on the right eye and $3+$ overaction of $\mathrm{IO}$ on the left eye. The examination revealed an exotropia of $45 \mathrm{PD}$ in the upgaze position, and $30 \mathrm{PD}$ in the downgaze position. The Bielschowsky head tilt test showed positive results on both sides [6]. A diagnosis of exotropia with bilateral superior oblique palsy was confirmed. We planned IO recession $8 \mathrm{~mm}$ on the right eye, and for the left eye, lateral rectus recession of $5 \mathrm{~mm}$, medial rectus resection of $4 \mathrm{~mm}$, and IO recession of $10 \mathrm{~mm}$ were planned. His pre-anesthetic history and examination were insignificant.

Copyright ( 12017 Yeungnam University College of Medicine

This is an Open Access article distributed under the terms of the Creative Commons Attribution Non-Commercial License (http://creativecommons.org/licenses/by-nc/4.0/) which permits unrestricted non-commercial use, distribution, and reproduction in any medium, provided the original work is properly cited. 

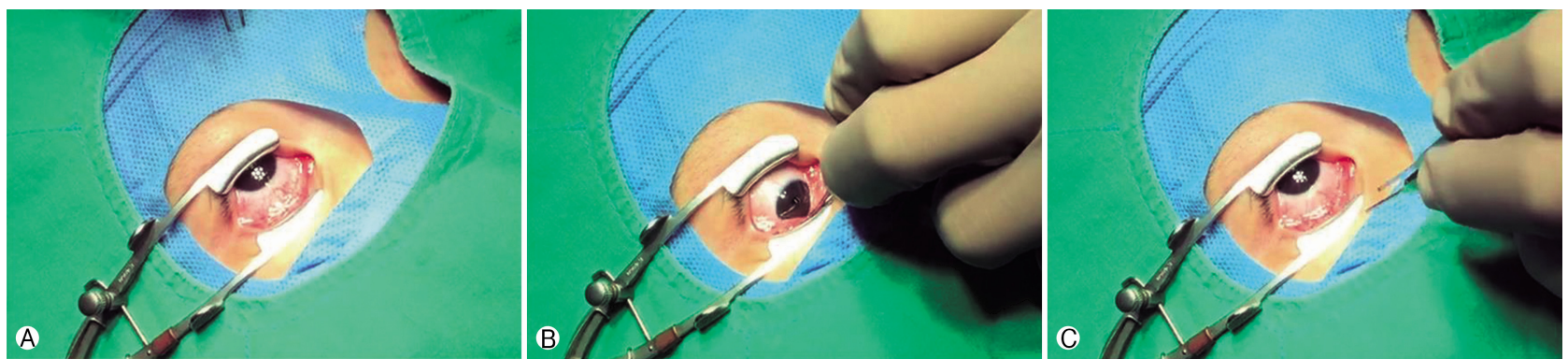

Fig. 1. Suddenly fixed upward ocular deviation occurred when the speculum was inserted into the right eye under general anesthesia (A). When the eyeball was pulled down using forceps, there was some resistance, such as contracture of the superior rectus (B). The eyeball sprang back into the upward position when the grasp was freed (C).

The patient received an intravenous administration of midazolam $0.05 \mathrm{mg} / \mathrm{kg}$ to reduce separation anxiety prior to the arrival in the operating room. Anesthesia was induced with intravenous Pentothal, Remifentanil, and Sevoflurane. The tracheal intubation was performed with non-depolarizing muscle relaxant (rocuronium $0.6 \mathrm{mg} / \mathrm{kg}$ ). General anesthesia was induced without complications. The surgical procedure of the left eye was performed initially without significant finding. On the other hand, suddenly fixed upward ocular deviation occurred when the speculum was inserted into the right eye (Fig. 1A). The left eye also showed the same phenomenon. When eyeball was pulled down using forceps, there was some resistance, such as contracture of superior rectus (Fig. 1B). The eyeball sprang back into the upward position when the grasp was freed (Fig. 1C). The traction suture, adjacent to the temporal limbus, was applied to fixate the globe into a good surgical field and to use an anatomical marker. An IO recession of $8 \mathrm{~mm}$ was performed via a fornix incision as planned. The patient was stable after recovery from general anesthesia.

\section{DISCUSSION}

The exact mechanism of this ocular change has not been fully elucidated to date. Previous study suggested that it may be due to the association between depth of anesthesia and ocular position [1]. For strabismus surgeons, the development of unexpected ocular deviation under general anesthesia can raise two questions: First, do I need to change the surgical plan? A previous study recommended that surgeons should rely on their preoperative measurements, despite the grossly different amount of deviation under general anesthesia than expected [3]. In the present case, the surgery was performed as planned preoperatively because both eyes showed the same upward deviation and the patient did not show any restrictive pattern at the preoperative measurement. The patient showed stable ocular alignment after the surgery. The second question is how this phenomenon should be prevented? Not only ophthalmic interventions, such as traction suture, but also anesthetic interventions should be considered to solve this problem. Rossiter et al. suggested the use of a muscle relaxant with a medium duration, such as atracurium or vecuronium [5]. We agree with their opinions. Conversely, the use of muscle relaxant requires a prolonged recover time because of the time to reverse the neuromuscular blockade. Other agents, such as rocuronium or cisatracurium, could be considered as alternative options with respect to the anesthetic perspective [7].

Suddenly fixed upward ocular deviation under general anesthesia can create both intraoperative difficulty and complications. If this occurs in a patient that required surgery at the superior rectus, the surgery is likely to be more difficult than usual. Surgeons needs to be aware of this possibility, and if it occurs, proceed with the surgery as planned preoperatively, and both ophthalmic and anesthetic interventions should be considered to solve this problem.

\section{CONFLICT OF INTEREST}

No potential conflict of interest relevant to this article was reported.

\section{ORCID}

Won Jae Kim, https://orcid.org/0000-0001-8869-0349

Sang-Jin Park, https://orcid.org/0000-0002-4838-2664

Myung Mi Kim, https://orcid.org/0000-0002-8564-1389 


\section{REFERENCES}

1. Ohmi E, Ogli K, Kani K. Eye position and electromyographic observation of squint eyes under general anesthesia. Ophthalmologica 1979;178:166-71.

2. Apt L, Isenberg S. Eye position of strabismus patients under general anesthesia. Am J Ophthalmol 1977;84:574-9.

3. Daien V, Turpin C, Lignereux F, Belghobsi R, Le Meur G, Lebranchu P, et al. Determinants of ocular deviation in esotropic subjects under general anesthesia. J Pediatr Ophthalmol Strabismus 2013;50:155-60.

4. Mims JL 3rd. "Double elevator palsy" eye supraducts during stage II general anesthesia supporting hypothesis of (supra) nuclear etiology. Binocul Vis Strabismus Q 2005;20:199-204.

5. Rossiter JD, Wood M, Lockwood A, Lewis K. Operating conditions for ocular surgery under general anaesthesia: an eccentric problem. Eye (Lond) 2006;20:55-8.

6. Wright KW, Strube YNJ. Pediatric ophthalmology and strabismus. 3rd ed. Oxford: Oxford University Press, 2012. p. 317-20.

7. Ortega A, Sarobe C, Iribarren MJ, Giráldez J. Cost analysis of neuromuscular blocking agents in the operating room: cisatracurium, atracurium, vecuronium and rocuronium. Pharm World Sci $2000 ; 22: 82-7$. 